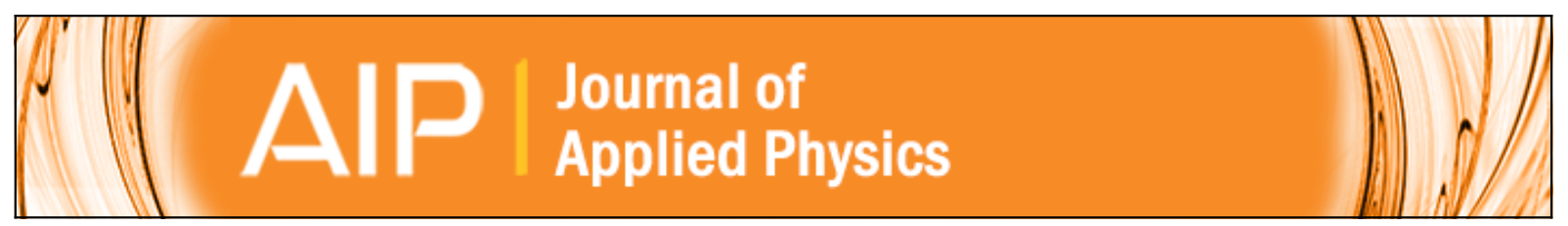

Thickness dependence of structure and piezoelectric properties at nanoscale of polycrystalline lead zirconate titanate thin films

E. B. Araújo, E. C. Lima, I. K. Bdikin, and A. L. Kholkin

Citation: Journal of Applied Physics 113, 187206 (2013); doi: 10.1063/1.4801961

View online: http://dx.doi.org/10.1063/1.4801961

View Table of Contents: http://scitation.aip.org/content/aip/journal/jap/113/18?ver=pdfcov

Published by the AIP Publishing

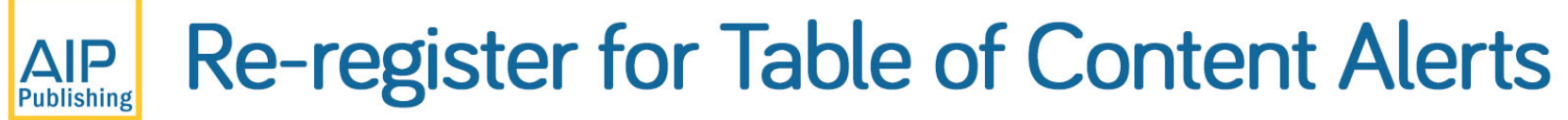




\title{
Thickness dependence of structure and piezoelectric properties at nanoscale of polycrystalline lead zirconate titanate thin films
}

\author{
E. B. Araújo, ${ }^{1, a)}$ E. C. Lima, ${ }^{1}$ I. K. Bdikin, ${ }^{2}$ and A. L. Kholkin ${ }^{3}$ \\ ${ }^{1}$ Faculdade de Engenharia de Ilha Solteira, UNESP - Univ Estadual Paulista, \\ Departamento de Física e Química, 15385-000 Ilha Solteira, SP, Brazil \\ ${ }^{2}$ Department of Mechanical Engineering and TEMA, University of Aveiro, 3810-193 Aveiro, Portugal \\ ${ }^{3}$ Department of Materials and Ceramic Engineering and CICECO, University of Aveiro, \\ 3810-193 Aveiro, Portugal
}

(Received 1 October 2012; accepted 3 January 2013; published online 8 May 2013)

\begin{abstract}
Lead zirconate titanate $\mathrm{Pb}\left(\mathrm{Zr}_{0.50} \mathrm{Ti}_{0.50}\right) \mathrm{O}_{3}$ (PZT) thin films were deposited by a polymeric chemical method on $\mathrm{Pt}(111) / \mathrm{Ti} / \mathrm{SiO}_{2} / \mathrm{Si}$ substrates to understand the mechanisms of phase transformations and the effect of film thickness on the structure, dielectric, and piezoelectric properties in these films. PZT films pyrolyzed at temperatures higher than $350{ }^{\circ} \mathrm{C}$ present a coexistence of pyrochlore and perovskite phases, while only perovskite phase grows in films pyrolyzed at temperatures lower than $300^{\circ} \mathrm{C}$. For pyrochlore-free PZT thin films, a small (100)-orientation tendency near the film-substrate interface was observed. Finally, we demonstrate the existence of a self-polarization effect in the studied PZT thin films. The increase of self-polarization with the film thickness increasing from $200 \mathrm{~nm}$ to $710 \mathrm{~nm}$ suggests that Schottky barriers and/or mechanical coupling near the film-substrate interface are not primarily responsible for the observed self-polarization effect in our films. C 2013 AIP Publishing LLC [http://dx.doi.org/10.1063/1.4801961]
\end{abstract}

\section{INTRODUCTION}

Lead zirconate titanate $\mathrm{Pb}\left(\mathrm{Zr}_{1-\mathrm{x}} \mathrm{Ti}_{\mathrm{x}}\right) \mathrm{O}_{3}(\mathrm{PZT})$ was probably one of the most studied ferroelectric materials in the past 50 years due to its excellent piezoelectric, pyroelectric, ferroelectric, and dielectric properties. ${ }^{1}$ The great potential of the PZT system in the thin film form has been demonstrated along past years for applications in capacitors, ${ }^{2}$ nonvolatile ferroelectric random memories, ${ }^{3}$ and many others.

PZT films produced by different physical and chemical methods exhibit different structural and electrical properties. Most studies have been conducted on oriented epitaxial PZT films to understand the size effects on their structural and electrical properties. ${ }^{4}$ Often, these studies have shown that the relaxation of misfit strain PZT films prepared as heterostructures or deposited on metallic substrates is accompanied by systematic changes in the polarization properties leading to an increase of the coercive field with decreasing thickness. ${ }^{5}$ These and others results suggest that the nature of the film-substrate interface, strain, microstructure, and other parameters play a significant role in the scaling down of ferroelectric thin films. In contrast to problems associated to downscaling of the solid-state electronics, some problems related to the synthesis of PZT thin films are periodically revisited providing a feedback to produce high performance devices.

The crystallization kinetics of PZT thin films is relatively well established for films prepared by rf-sputtering, ${ }^{6}$ laser ablation, ${ }^{7}$ and sol-gel. ${ }^{8}$ Alternatively, ferroelectric thin films have been prepared by polymer precursor technique based on Pechini method. ${ }^{9}$ In general, the crystallization of

\footnotetext{
${ }^{\text {a) }}$ Author to whom correspondence should be addressed. Electronic mail: eudes@dfq.feis.unesp.br. Fax: +55 18 3742-4868.
}

PZT films using low temperature deposition techniques occurs from the amorphous structure that first transforms into an intermediate non-ferroelectric phase and further transforms into the perovskite phase after an adequate pyrolysis and thermal annealing. Naturally, this transformation kinetic depends on the method used to prepare ferroelectric thin films. This undesirable non-ferroelectric phase is often described as a pyrochlore, sometimes also referred as $\mathrm{Pb}$ deficient fluorite phase. ${ }^{10}$ The presence of this phase degrades the most important ferroelectric properties of the film and consequently must be avoided during the crystallization. Although different studies were conducted on PZT films produced by polymer precursor method, ${ }^{11}$ up to now the kinetics of growth of the perovskite phase in these films is not yet systematically studied, as well the thickness effect on the structure, dielectric, and piezoelectric properties of these films.

The purpose of the present work is to report the phase transformations and the thickness effect on the structure, dielectric, and piezoelectric properties of polycrystalline PZT thin films with no preferential orientation, prepared by polymeric chemical method. Furthermore, the existence of a self-polarization effect is shown in these films, and its dependence on the film's thickness sheds light on the mechanism of self-polarization phenomenon.

\section{EXPERIMENTAL}

PZT films with a nominal composition of $\mathrm{Pb}\left(\mathrm{Zr}_{0.50} \mathrm{Ti}_{0.50}\right) \mathrm{O}_{3}$ were deposited by spin coating on $\mathrm{Pt}(111) / \mathrm{Ti} / \mathrm{SiO}_{2} / \mathrm{Si}$ substrates using a chemical polymeric method described elsewhere. ${ }^{11}$ To compensate the loss of lead during the film crystallization and to stabilize the growth of the perovskite phase, $10 \mathrm{~mol}$. \% of lead oxide was added in excess 
of the nominal composition. To remove organics, the films were heat annealed at a pyrolysis temperature between 150 and $450^{\circ} \mathrm{C}$ for $30 \mathrm{~min}$ and finally crystallized at $700^{\circ} \mathrm{C}$ for $1 \mathrm{~h}$. The thicknesses of the final films were between 200 and $710 \mathrm{~nm}$.

X-ray diffraction (XRD) patterns of the PZT films were obtained using a Rigaku Ultima IV diffractometer with $\mathrm{CuK}_{\alpha}(1.5406 \AA)$ radiation. To study the structure of the films in depth profile, XRD measurements were performed at room temperature in step scan mode using the glancing incidence configuration at a fixed $\theta$ angle from $2^{\circ}$ to $6^{\circ}$ and scanning the $2 \theta$ angle from $20^{\circ}$ to $60^{\circ}$. All lattice parameters were obtained from Rietveld refinements. For Rietveld ${ }^{12}$ analysis, XRD data were input into the GSAS ${ }^{13}$ structure refinement code under the EXPGUI ${ }^{14}$ interface. Peak profiles were fitted using the Thompson-Cox-Hastings ${ }^{15}$ pseudoVoigt function while a sixth-order polynomial was used to fit the background. For refinements, a pure tetragonal phase with $P 4 m m$ space group was considered, where the $P b^{2+}$ ion occupies sites at $(0,0,0), \mathrm{Ti}^{4+} / \mathrm{Zr}^{4+}$ and $O_{I}^{2-}$ occupy sites (1/ $2,1 / 2, \mathrm{z})$, and $O_{I I}^{2-}$ occupy sites at $(1 / 2,0, \mathrm{z})$. The main criteria for judging on the quality of the fitting is the final fit of the calculated pattern compared to the observed data, but the Rfactors weighted profile $w R_{p}$, the statistically expected $R_{\text {exp }}$, and the goodness-of-fit indices $\chi^{2}=w R_{p} / R_{\exp }$ are usually used to attest the quality of a fit. ${ }^{16}$ Williamson-Hall analysis was applied to evaluate the microstrain $(\Delta d / d)$ and crystallite size of the PZT films according to the formula $\Gamma \cos \theta=\lambda / D+(\Delta d / d) 4 \operatorname{sen} \theta$, where $d$ is the lattice spacing, $\Gamma$ is the full width at half maximum (FWHM) value due to peak broadening, $\lambda$ is the wavelength, $\theta$ is the Bragg angle, and $D$ is the average grain size, respectively.

The piezoresponse of the films was obtained by the modified commercial atomic force microscope (AFM) (Multimode, Nanoscope III, Bruker and Ntegra Prima, NTMDT). N-doped Si tips were used with a spring constant $42 \mathrm{~N} / \mathrm{m}$ to avoid possible electrostatic effects. To measure electrical properties, several gold electrodes $(0.3 \mathrm{~mm}$ in diameter) were deposited over an area of $1 \times 1 \mathrm{~cm}^{2}$ on the films through a mask to form capacitors. Dielectric permittivity measurements were performed using an Agilent 4284A LCR meter at $10 \mathrm{kHz}$. A modified Sawyer-Tower circuit at $20 \mathrm{~Hz}$ was used to measure the polarization hysteresis loops.

\section{RESULTS AND DISCUSSION}

Figure 1(a) shows XRD profiles of PZT thin films prepared under different pyrolysis temperature. In this figure, the indexed $(h \mathrm{kl})$ peaks were associated to the tetragonal phase of the PZT, while both (222) and (400) peaks at $2 \theta=29.8^{\circ}$ and $35.0^{\circ}$ were attributed to the pyrochlore phase. The XRD profiles of the PZT films pyrolyzed between $350{ }^{\circ} \mathrm{C}$ and $450^{\circ} \mathrm{C}$ show a coexistence of the pyrochlore and perovskite phases, while for films pyrolyzed at $150^{\circ} \mathrm{C}$ and $300^{\circ} \mathrm{C}$ only peaks associated with the perovskite phase were observed. The presence of the (222) and (400) reflections in Fig. 1(a) suggests a pyrochlore type phase of the $\mathrm{Pb}_{2} \mathrm{Ti}_{2} \mathrm{O}_{6}$ structure, with the possibility that $\mathrm{Zr}$ ions are able to
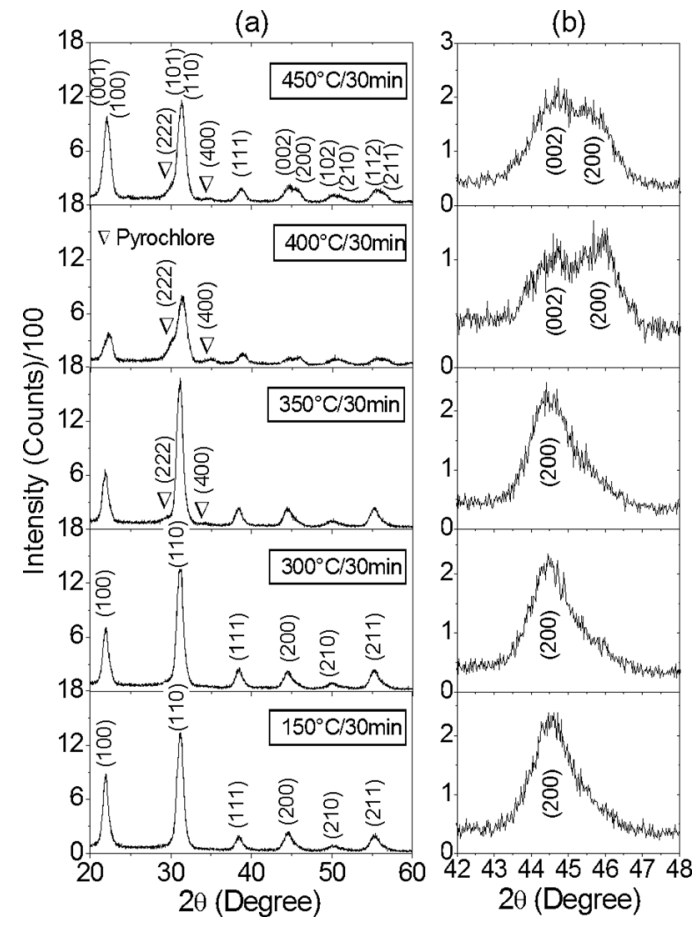

FIG. 1. (a) XRD profiles of PZT thin films crystallized at $700{ }^{\circ} \mathrm{C}$ for $1 \mathrm{~h}$ on $\mathrm{Pt}(111) / \mathrm{Ti} / \mathrm{SiO}_{2} / \mathrm{Si}$ substrates and pyrolyzed at different temperatures. All XRD patterns were recorded at a fix glancing angle $\theta=6^{\circ}$. (b) XRD profiles around the (002) and (200) reflections for PZT films.

substitute for $\mathrm{Ti}$ ions in the pyrochlore structure to form a more realistic metastable $\mathrm{Pb}_{2}\left(\mathrm{Zr}_{1-\mathrm{x}} \mathrm{Ti}_{\mathrm{x}}\right)_{2} \mathrm{O}_{6}$ structure. ${ }^{17}$ In the literature, there are several reports on the possible composition of the pyrochlore phase for PZT films prepared by sol-gel route. ${ }^{18,19}$ In general, these studies were centered on $\mathrm{Pb}\left(\mathrm{Zr}_{0.53} \mathrm{Ti}_{0.47}\right) \mathrm{O}_{3}$ film composition for historical reasons, and there is a consensus that above mentioned (222) and (400) reflections can be attributed to the pyrochlore $\mathrm{Pb}_{2}\left(\mathrm{Zr}_{0.53} \mathrm{Ti}_{047}\right)_{2} \mathrm{O}_{6}$ phase. Our study, however, was centered on the $\mathrm{Pb}\left(\mathrm{Zr}_{0.50} \mathrm{Ti}_{0.50}\right) \mathrm{O}_{3}$ composition, and this approach offers some advantages to interpret the formation of pyrochlore phase in our films, as we shall see in the next paragraph.

Figure 1(b) shows more closely the evolution of the (002) and (200) peaks in XRD patterns of the PZT films with different pyrolysis temperatures. For films pyrolyzed at $400{ }^{\circ} \mathrm{C}$ and $450{ }^{\circ} \mathrm{C}$ a clear structure of two peaks was observed at $2 \theta=44.7^{\circ}$ and $45.9^{\circ}$. However, a structure of a single broad peak was observed in XRD patterns of the films pyrolyzed at temperatures below $300^{\circ} \mathrm{C}$. These remarkable changes give us an important qualitative information about the structure of the PZT films and indirectly infer about the kinetics of the pyrochlore phase and its tentative composition.

For PZT ceramics, the splitting of $(00 l)$ and $(h 00)$ reflections in XRD patterns with decreasing $\mathrm{Zr} / \mathrm{Ti}$ ratio is a clear evidence that the symmetry changes from rhombohedral to tetragonal. ${ }^{20}$ The substitution of $\mathrm{Zr}$ with $\mathrm{Ti}$ ions in $\mathrm{PbTiO}_{3}$ leads to a change in the structure from tetragonal to rhombohedral symmetry and vice versa for substitution of $\mathrm{Ti}$ ions with $\mathrm{Zr}$ ions. As the splitting of $(00 l)$ and $(h 00)$ reflections is 
more evident for higher $2 \theta$ angles in XRD pattern, we focus our discussions on the (002) and (200) reflections. In other words, PZT rhombohedral compositions (Zr-rich) exhibit a single (200) reflection while tetragonal compositions (Tirich) exhibit double (002) and (200) reflections in the XRD pattern. Supported by the above discussions it is clear in Figure 1(b) that different pyrolysis temperature is favorable to produce PZT thin films with distinct crystalline structure.

The splitting of (002) and (200) reflections in Figure 1(b) for PZT films pyrolyzed at $400{ }^{\circ} \mathrm{C}$ and $450{ }^{\circ} \mathrm{C}$ clearly indicates a shift toward the tetragonal side in the composition-temperature phase diagram of the PZT system. ${ }^{1}$ This implies a Ti-rich PZT composition $(\mathrm{Zr} / \mathrm{Ti}<1)$ in comparison to the nominal $\mathrm{Pb}\left(\mathrm{Zr}_{0.50} \mathrm{Ti}_{0.50}\right) \mathrm{O}_{3}$ composition $(\mathrm{Zr} /$ $\mathrm{Ti}=1$ ), which would enrich the pyrochlore phase and reduce the perovskite phase in $\mathrm{Zr}$ ions. These results indicate that the growth of a $\mathrm{Zr}$-rich pyrochlore phase $\mathrm{Pb}_{2}\left(\mathrm{Zr}_{1-\mathrm{x}} \mathrm{Ti}_{\mathrm{x}}\right)_{2} \mathrm{O}_{6}$ $(\mathrm{Zr} / \mathrm{Ti}>1)$ is more energetically favorable for films pyrolyzed at $400{ }^{\circ} \mathrm{C}$ and $450{ }^{\circ} \mathrm{C}$. However, a result slightly different was observed in Figure 1(b) for PZT films pyrolyzed at 150,300 , and $350^{\circ} \mathrm{C}$. For these films, the structure of a single broad (200) peak was observed around $2 \theta=45.5^{\circ}$, which is an expected characteristic of the tetragonal phase for the studied PZT with $\mathrm{Zr} / \mathrm{Ti}=1$. While PZT films pyrolyzed at $150^{\circ} \mathrm{C}$ and $300^{\circ} \mathrm{C}$ are pyrochlore-free, the film pyrolyzed at $350^{\circ} \mathrm{C}$ presents a small amount of pyrochlore phase, as indicated by the presence of both (222) and (400) peaks in Figure 1(a). In this case, we can infer the growth of a pyrochlore and perovskite phases both having $\mathrm{Zr} / \mathrm{Ti} \cong 1$ in the film pyrolyzed at $350{ }^{\circ} \mathrm{C}$ for $30 \mathrm{~min}$.

Based on the results shown in Figure 1(a) and discussion in the above paragraph, the pyrolysis at $300^{\circ} \mathrm{C}$ for $30 \mathrm{~min}$ followed by a final crystallization at $700{ }^{\circ} \mathrm{C}$ for $1 \mathrm{~h}$ is established as an optimal condition to prepare pyrochlore-free PZT films in the present work. Results based on the XRD analysis as a function of glancing $\theta$ angle are shown in Figure 2 for pyrochlore-free PZT film with $710 \mathrm{~nm}$ in thickness. These analyzes give us a qualitative behavior of each studied parameter in the depth profile, where the lattice parameters were obtained from Rietveld refinements. The microstrain $(\Delta d / d)$ and the crystallite size (D) obtained from Williamson-Hall analysis are shown in Figures 2(a) and 2(b), respectively. These results demonstrated a non-uniform microstrain and crystallite size along the film thickness, where both parameters increase almost linearly from top surface to the bottom film-substrate interface. The obtained microstrain $(0.56 \%-0.79 \%)$ of the studied film was similar to values reported in the literature for polycrystalline $\mathrm{Nd}$ modified PZT films ${ }^{21}$ and slightly larger than values reported for (100)-oriented PZT films prepared by rf-sputtering technique $\left(\sim 0.15 \%\right.$ for film with $100 \mathrm{~nm}$ in thickness). ${ }^{22}$ On the other hand, the values obtained for the crystallite size $(18-41 \mathrm{~nm})$ were lower than those values reported in the literature ( $\sim 90 \mathrm{~nm}$ for film with $270 \mathrm{~nm}$ in thickness) for PZT films prepared by rf-sputtering. ${ }^{23}$

The strain in ferroelectric thin films is normally attributed to the sum of the elastic strain due to the lattice difference between the film and the substrate, the thermal strain as the substrate imposes its thermal evolution onto the film, and

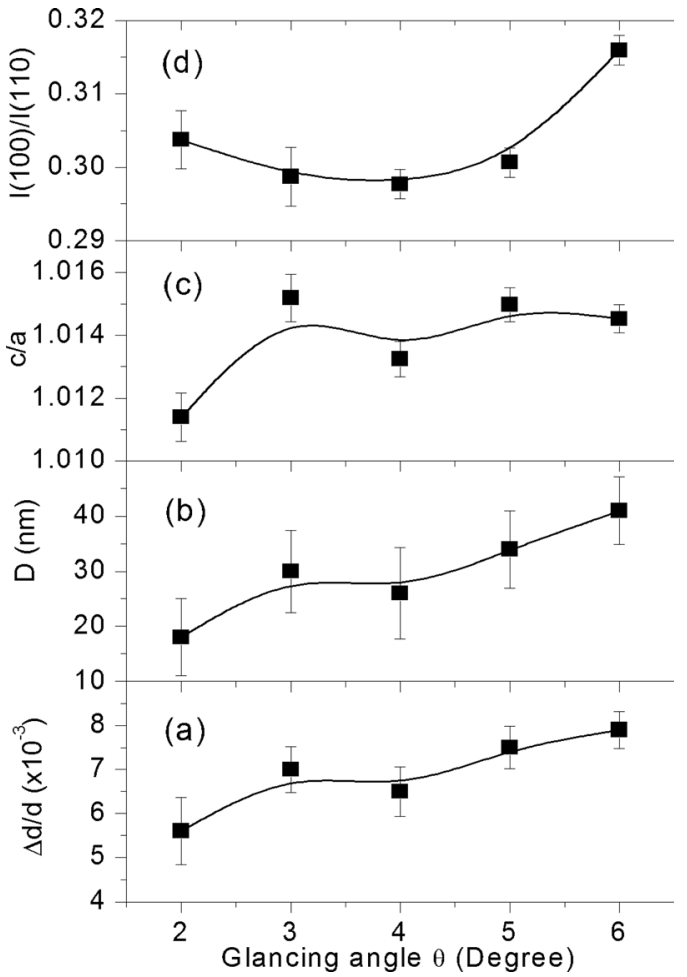

FIG. 2. (a) Microstrain, (b) crystallite size, (c) tetragonality factor $c / a$, and (d) $\mathrm{I}(100) / \mathrm{I}(110)$ ratio as a function of XRD glancing angle $\theta$ for pyrochlorefree PZT thin film deposited on $\mathrm{Pt}(111) / \mathrm{Ti} / \mathrm{SiO}_{2} / \mathrm{Si}$ substrate, pyrolyzed at $300^{\circ} \mathrm{C}$ for $30 \mathrm{~min}$, and crystallized at $700^{\circ} \mathrm{C}$ for $1 \mathrm{~h}$.

the spontaneous transformation strain linked to the phase transitions. ${ }^{24}$ In this work, Figure 2(a) indicates a microstrain relaxation from the bottom film-substrate interface to the top surface. Furthermore, Figure 2(b) shows that close to the film-substrate interface the crystallite size are slightly larger than near the top film surface.

Figure 2(c) shows the behavior of the tetragonality factor $(c / a)$ as a function of the glancing angle $\theta$. In this figure we observe that the $c / a$ remains almost constant for $3^{\circ} \leq \theta \leq 6^{\circ}$ and decreases slightly to $c / a=1.011$ near the film top surface. Figure 2(d) presents the behavior of the maximum peak intensities $\mathrm{I}(100) / \mathrm{I}(110)$ ratio as a function of glancing incidence angle $\theta$. By increasing the $\theta$ angle, it is expected that this ratio should be constant for a homogeneous and stress-free structure along the film thickness. The $\mathrm{I}(100) / \mathrm{I}(110)$ ratio remains almost unchanged for $\theta$ smaller than $5^{\circ}$ but increases by $5 \%$ for $\theta=6^{\circ}$. This increasing is a result of the texture changes in the film depth and indicates a small (100) orientation tendency near the film-substrate interface. The texture for PZT thin films is generally influenced by several factors such as pyrolysis temperature ${ }^{36}$ or seeding layers ${ }^{37}$ introduced during processing, leading to (111) or (100) PZT orientation of the film grown on (111) Pt metallic electrode. The slight tendency to (100) texture observed in the present work at the film-substrate interface indicates that a small amount of nuclei of (100) orientation is formed at the film-substrate interface during pyrolysis of the first deposited layer. These nuclei became unstable on the next deposited layers due to lower interfacial energy at the film-substrate interface. Consequently, a randomly oriented film grew on the partially (100) oriented layer. 


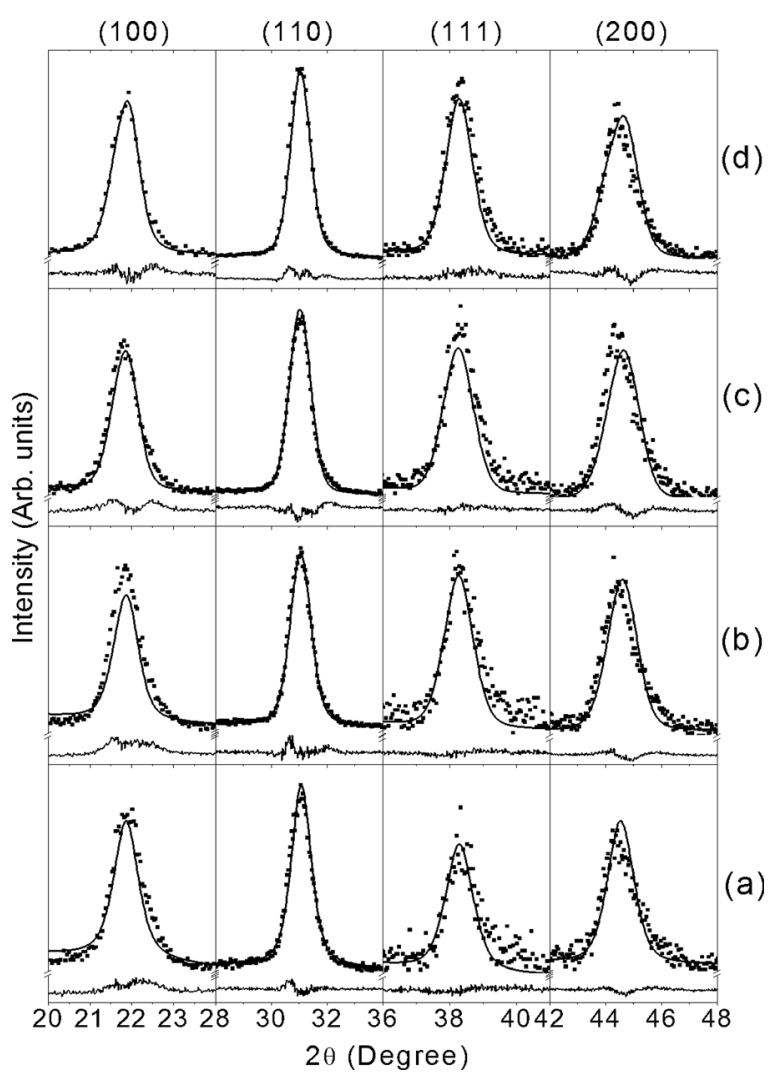

FIG. 3. The observed (dots), calculated (lines), and different (bottom lines) profiles of selected (100), (110), (111), and (200) tetragonal reflections for pyrochlore-free PZT thin films with different thicknesses: (a) $200 \mathrm{~nm}$, (b) $380 \mathrm{~nm}$, (c) $500 \mathrm{~nm}$, and (d) $710 \mathrm{~nm}$.

Figure 3 depicts the observed, calculated, and different profiles of selected (100), (110), (111), and (200) reflections of pyrochlore-free PZT thin films with different thicknesses. The refined cell parameters and R-factors of PZT films with different thicknesses were summarized in Table I. The obtained $\chi^{2}$ values (between 2.3 and 3.3) are slightly higher than the observed for bulk ceramics. ${ }^{25}$ The main reasons for these differences can be associated to larger line width and effects of substrates in thin films, if compared with bulk ceramics or single crystals.

Figure 4 shows the AFM micrographs of surface topography of PZT films of different thicknesses. Analysis of AFM data indicated that the PZT films present a homogeneous and crack-free surface. The obtained average grain size were $72,73,74$, and $77 \mathrm{~nm}$ for films of thickness 200,380 , 500 , and $710 \mathrm{~nm}$, respectively. The grain size increases slightly with increasing film thickness. Based on this result,

TABLE I. Refined cell parameters and R-factors of PZT films with different thicknesses.

\begin{tabular}{lcccccc}
\hline \hline & \multicolumn{2}{c}{ Cell parameters } & & \multicolumn{3}{c}{ R-factors } \\
\cline { 2 - 3 } \cline { 5 - 7 } Film thickness & $a(\AA)$ & $c(\AA)$ & & $\chi^{2}$ & $\mathrm{R}_{\mathrm{wp}}$ & $\mathrm{R}_{\text {exp }}$ \\
\hline $200 \mathrm{~nm}$ & 4.0774 & 4.0966 & & 2.3 & 17.1 & 13.3 \\
$380 \mathrm{~nm}$ & 4.0670 & 4.1114 & & 3.3 & 17.6 & 13.6 \\
$500 \mathrm{~nm}$ & 4.0547 & 4.1113 & & 3.1 & 15.9 & 12.1 \\
$710 \mathrm{~nm}$ & 4.0692 & 4.1301 & & 3.2 & 15.2 & 11.2 \\
\hline \hline
\end{tabular}

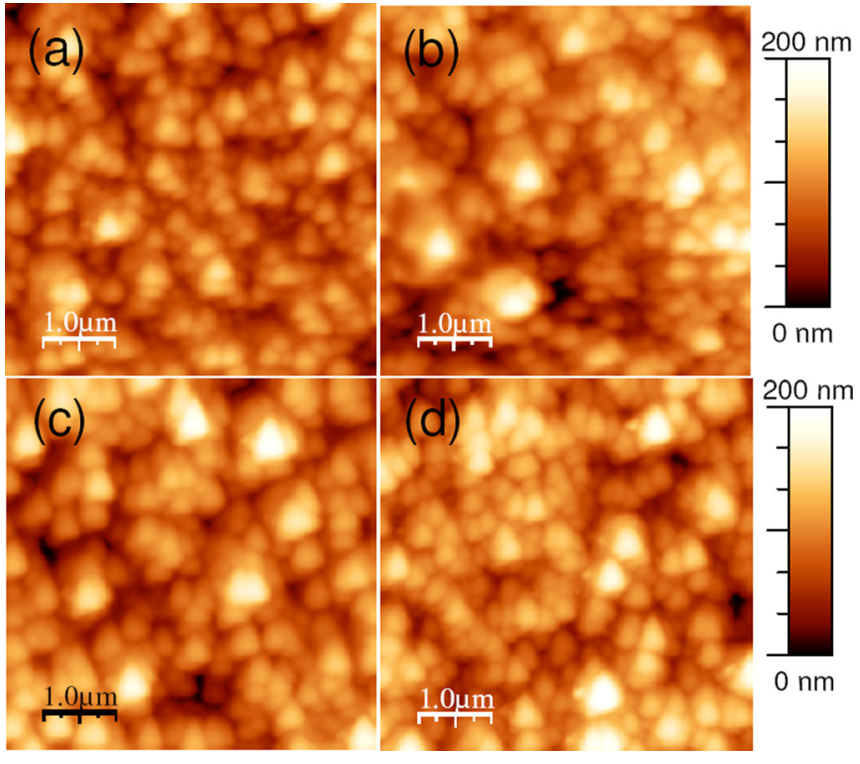

FIG. 4. AFM images $\left(5 \times 5 \mu \mathrm{m}^{2}\right)$ of pyrochlore-free PZT thin films with different thicknesses: (a) $200 \mathrm{~nm}$, (b) $380 \mathrm{~nm}$, (c) $500 \mathrm{~nm}$, and (d) $710 \mathrm{~nm}$.

we can conclude that grain sizes of the films are essentially the same for different thicknesses. The obtained RMS roughness was $24 \mathrm{~nm}$ for the film with $200 \mathrm{~nm}$ in thickness and $31 \mathrm{~nm}$ for films of thickness 380,500 , and $710 \mathrm{~nm}$.

Thickness dependence of the structural parameters of PZT thin films was also studied from XRD data recorded at a fix glancing angle $\theta=6^{\circ}$. Figures $5(\mathrm{a})$ and $5(\mathrm{~b})$ show the microstrain and crystallite size of PZT films plotted as a function of the film thickness. In these figures, we can see a

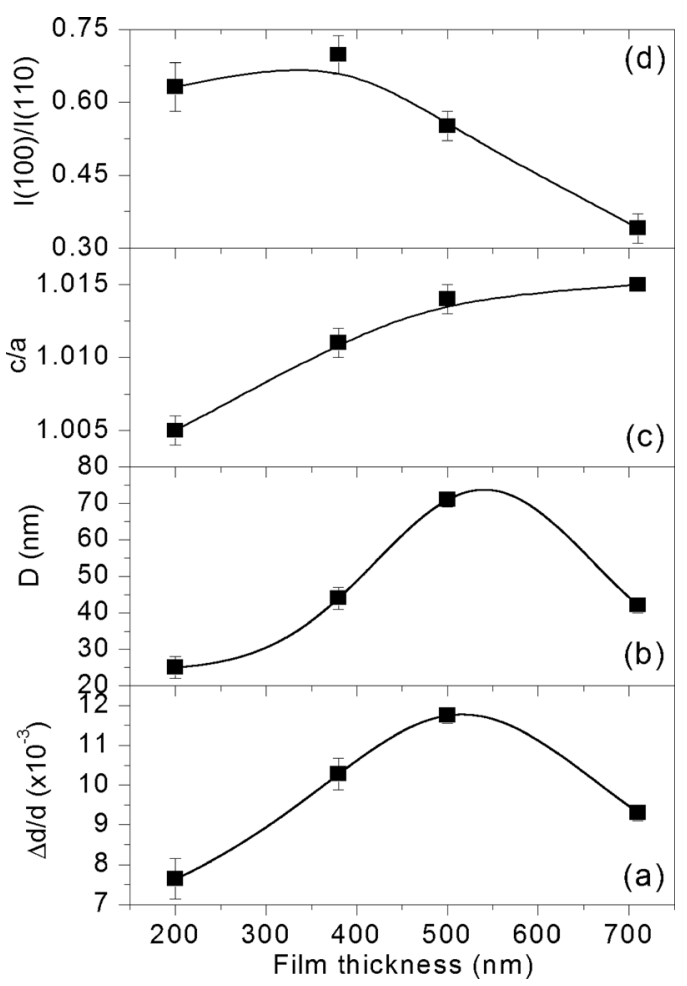

FIG. 5. (a) Microstrain, (b) crystallite size, (c) tetragonality factor $c / a$, and (d) $\mathrm{I}(100) / \mathrm{I}(110)$ ratio of pyrochlore-free PZT thin films as a function of film thickness. 
maximum at $1.17 \%$ and $71 \mathrm{~nm}$ for microstrain and crystallite size, respectively, for the film with $500 \mathrm{~nm}$ in thickness. Figure 5(c) plots the tetragonality factor $c / a$ as a function of film thickness. The lattice parameters obtained from Rietveld refinements lead to $c / a=1.015$ for the film with $710 \mathrm{~nm}$ in thickness. The tetragonality factor was also measured for films with different thicknesses to show a decreasing to $c / a=1.005$ for the film with $200 \mathrm{~nm}$ in thickness, following the non-linear behavior shown in Figure 5(c). Finally, the I(100)/I(110) ratio is plotted as a function of the film thickness in Figure 5(d). This ratio was 0.34 for the film of $710 \mathrm{~nm}$ in thickness and increases to 0.63 when the film thickness decreases to $200 \mathrm{~nm}$. This result suggests a (100)-orientation tendency for thinner films studied in the present work.

Microstrain and crystallite size seems to follow the film (100)-orientation tendency so that it is minimized to $0.76 \%$ and $25 \mathrm{~nm}$, respectively, for films with $200 \mathrm{~nm}$ thick. A similar tendency of the microstrain decreasing with film thickness decreasing was observed for PZT films prepared by pulsed laser deposition technique. ${ }^{21}$ The observed behavior for the microstrain and crystallite size in our films are most probably associated to the residual stress, since films with different thicknesses show essentially the same microstructure with grain size ranged between 72 and $77 \mathrm{~nm}$ and a minimum unit cell volume at $67.6 \AA^{3}$ at the film with $500 \mathrm{~nm}$ in thickness.

Figure 6(a) shows the thickness dependence of the real $\left(\varepsilon^{\prime}\right)$ and imaginary $\left(\varepsilon^{\prime \prime}\right)$ dielectric permittivity at $20 \mathrm{kHz}$ frequency for the PZT films with different thicknesses. The $\varepsilon^{\prime}$ values increase almost linearly from 258 to 463 as the film thickness increases from $200 \mathrm{~nm}$ to $710 \mathrm{~nm}$, while $\varepsilon^{\prime \prime}$ remains almost constant within the same thickness range. This result agrees with several reports for PZT thin films obtained by different
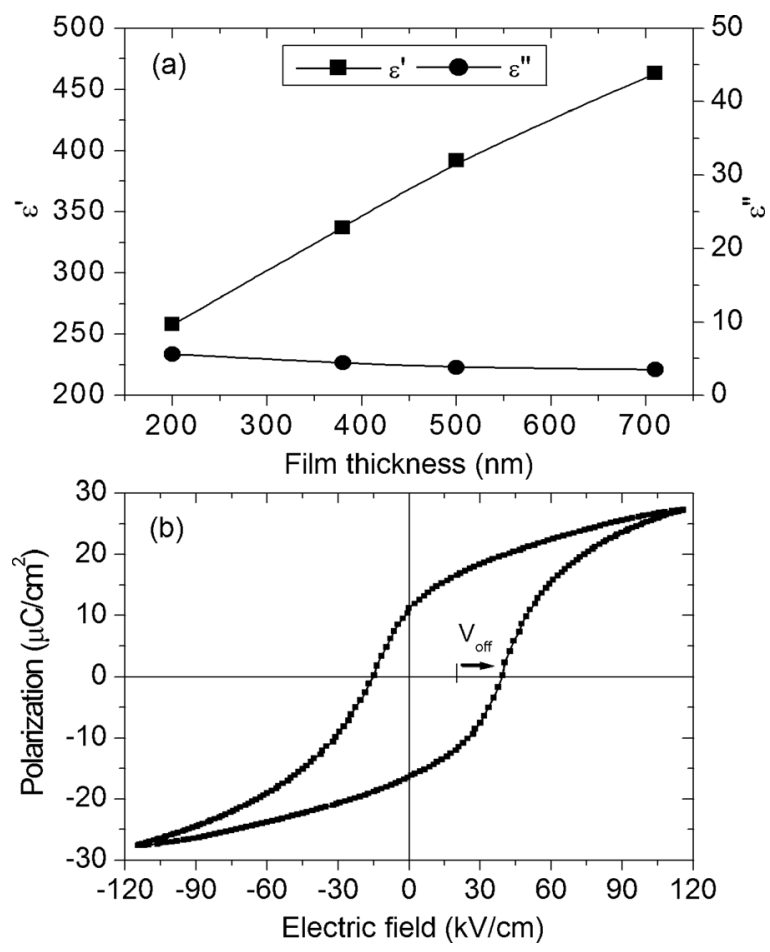

FIG. 6. (a) Real $\left(\varepsilon^{\prime}\right)$ and imaginary $\left(\varepsilon^{\prime \prime}\right)$ dielectric permittivity of PZT films with different thicknesses, recorded at $10 \mathrm{kHz}$ frequency. (b) Hysteresis loops of the PZT film with $710 \mathrm{~nm}$ in thickness, recorded at $20 \mathrm{~Hz}$. chemical routes. The behavior observed in Figure 6(a) for dielectric permittivity can be understood in terms of intrinsic and extrinsic contributions. ${ }^{26}$ The main intrinsic contributions are mechanical stresses, grain size, and film orientation, while $180^{\circ}$ domain wall motion is one of the most important extrinsic contributions. As the PZT films studied in this work present essentially the same grain size for different thickness, the extrinsic contributions are probably the main factors responsible for the behavior shown in Figure 6(a). The hysteresis loops of the PZT film with $710 \mathrm{~nm}$ thickness are shown in Figure 6(b). In this figure, the remnant polarizations are $P_{r}^{+}=+11.2 \mu \mathrm{C} /$ $\mathrm{cm}^{2}$ and $P_{r}^{-}=-16.1 \mu \mathrm{C} / \mathrm{cm}^{2}\left(\Delta P=-4.9 \mu \mathrm{C} / \mathrm{cm}^{2}\right)$ while the coercive electric fields are $E_{c}^{+}=+39.5 \mathrm{kV} / \mathrm{cm}$ and $E_{c}^{-}$ $=-14.6 \mathrm{kV} / \mathrm{cm}$. The observed internal bias field is reflected by the apparent shift towards the positive electric fields in Figure 6(b), which is a signature of the macroscopic selfpolarization effect in this film.

Figure 7 shows the vertical piezoresponse force microscopy (VPFM) images of PZT thin films with different thicknesses $(200-710 \mathrm{~nm})$. The out-of-plane components of polarization were obtained by detecting vertical vibrations of the piezoresponse force microscopy (PFM) cantilever. In these figures, a dc voltage of $+30 \mathrm{~V}$ was applied in the white area while a voltage of $-30 \mathrm{~V}$ was applied in the dark area. The external areas designate the film before poling. Based on the figures, the distribution of the local piezoresponse signal before and after poling could be constructed as a function of film thickness, as shown below.

Figure 8(a) compares the distribution of the local piezoresponse signal before and after poling for the PZT film with $710 \mathrm{~nm}$ thick. Only one peak shifting to negative $d_{33}$ values before poling in Figure 8(a) is also an indicative of the local self-polarization effect at the nanoscale. The two peaks at positive and negative $d_{33}$ values in this figure are associated with fully reoriented polar states and can be considered as

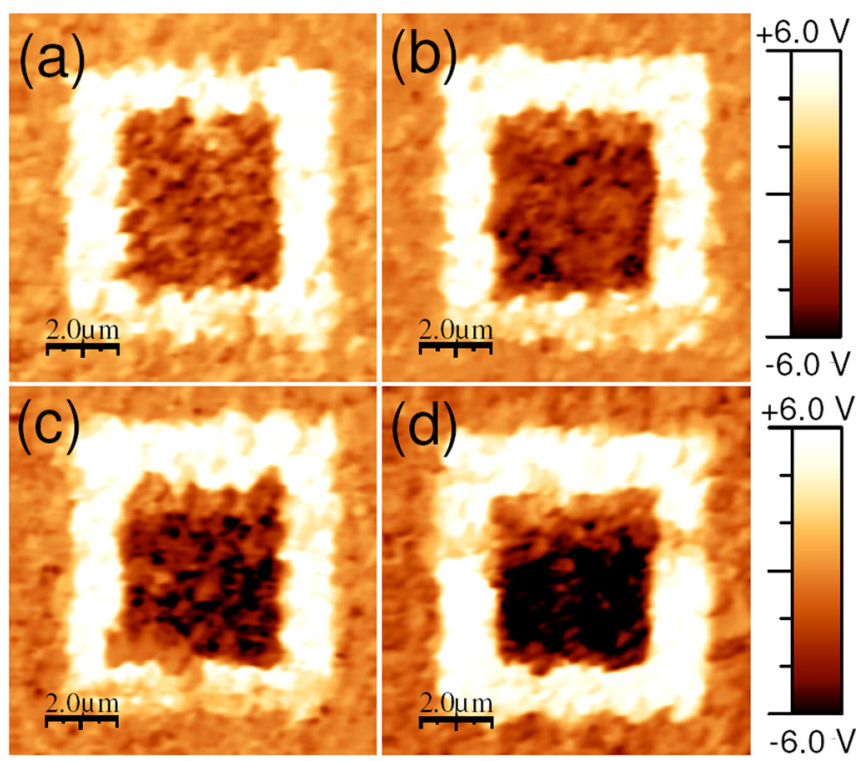

FIG. 7. VPFM images of $10 \times 10 \mu \mathrm{m}^{2}$ area of pyrochlore-free PZT thin films with different thicknesses: (a) $200 \mathrm{~nm}$, (b) $380 \mathrm{~nm}$, (c) $500 \mathrm{~nm}$, and (d) $710 \mathrm{~nm}$. All films were deposited on $\mathrm{Pt}(111) / \mathrm{Ti} / \mathrm{SiO}_{2} / \mathrm{Si}$ substrates, pyrolyzed at $300^{\circ} \mathrm{C}$ for $30 \mathrm{~min}$, and crystallized at $700^{\circ} \mathrm{C}$ for $1 \mathrm{~h}$. 

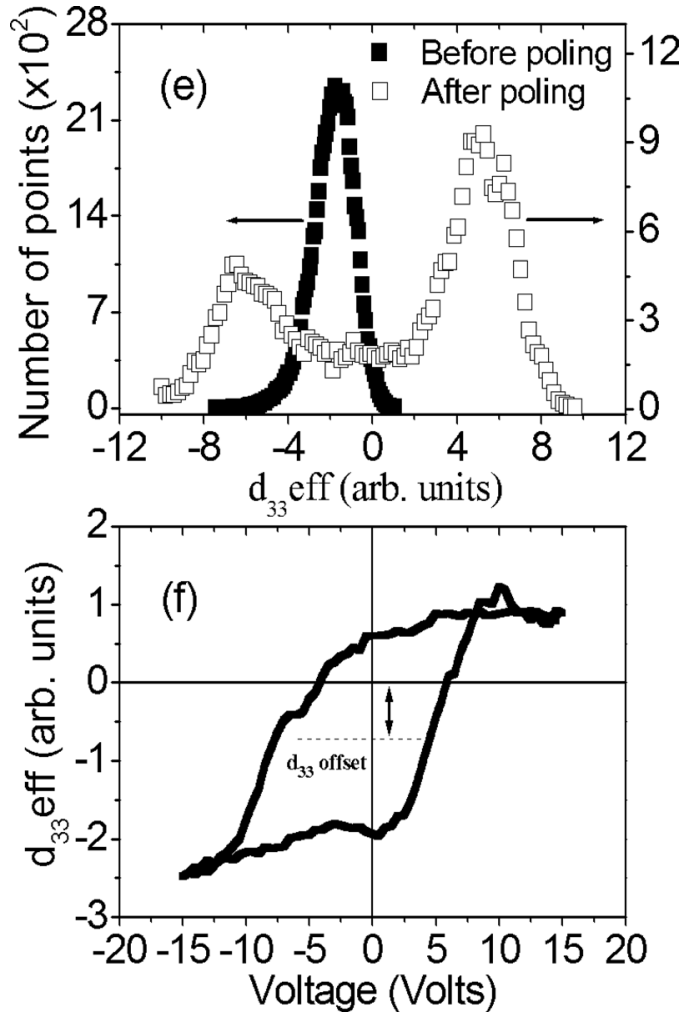

FIG. 8. (a) Distribution of the local piezoresponse before and after poling and (b) local piezoresponse hysteresis loops for the PZT thin film with $710 \mathrm{~nm}$ in thickness.

effective values of the intrinsic piezoresponse inside a single domain region. Finally, the local piezoresponse hysteresis loop is shown in Figure 8(b). The obtained local $d_{33}$ values and coercive voltages in this figure were similar to other reports in the literature. Again, the loop is slightly asymmetric due to self-polarization effect in the studied PZT film. Therefore, the shift observed both at macro- and nanoscales, as shown in Figures 6(b), 8(a), and 8(b), indicates that the observed voltage offset cannot be a spurious effect associated with a partial polarization frozen in one direction of the film but attest the existence of a self-polarization effect in the studied film.

Figure 9(a) shows the distribution of the local piezoresponse before poling for PZT films with different thicknesses while distributions after poling for same films are shown in Figure 9(b). The behavior of the self-polarization $P_{i}$ as a function of film thickness is shown in Figure 9(c). In this figure, $P_{i}$ presents essentially the same value for films with thicknesses between $200 \mathrm{~nm}$ and $380 \mathrm{~nm}$ but increases almost linearly for negative values from $500 \mathrm{~nm}$ up to $710 \mathrm{~nm}$ in thickness. The $P_{i} \propto d_{33} / \varepsilon^{\prime}$ relation provides a qualitative description for the self-polarization effect in our PZT films, ${ }^{27}$ where $d_{33}$ is the maximum piezoresponse distribution before poling in Figure 9(a) and $\varepsilon^{\prime}$ is the real part of the dielectric permittivity of the respective film in Figure 6(a). This relation is reasonable if we consider that the coupling between the longitudinal piezoelectric coefficient $\left(d_{33}\right)$ and the dielectric permittivity $\left(\varepsilon^{\prime}\right)$ and polarization $(P)$ can be described by the equation $d_{33}$ $=2 Q_{11} \varepsilon_{0} \varepsilon^{\prime} P$, where $Q_{11}$ is the electrostriction coefficient and $\varepsilon_{0}$ is the dielectric permittivity of vacuum. In the latter
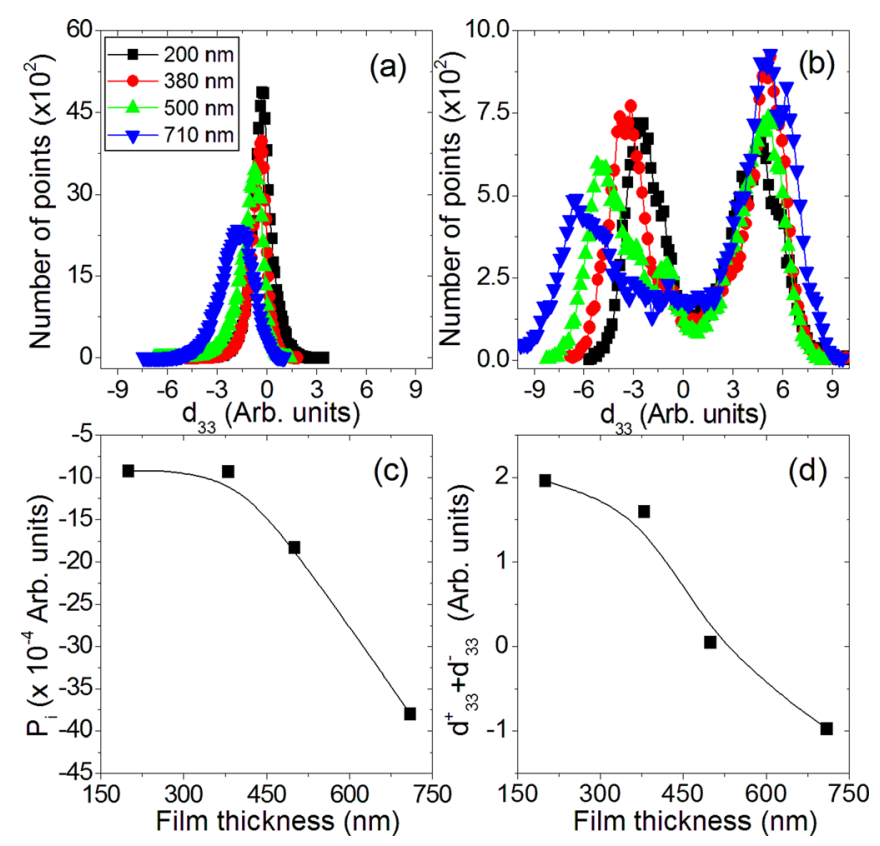

FIG. 9. Distribution of the local piezoresponse of PZT films with different thicknesses: (a) before poling and (b) after poling. (c) Self-polarization $\left(\mathrm{P}_{\mathrm{i}}\right)$ of PZT films as a function of thickness and (d) difference for the maximum $d_{33}^{+}-d_{33}^{-}$as after poling for different thicknesses. Lines are drawn as a guide to the eye.

equation, we assume that the effective $Q_{11}$ remains almost constant since the domain structure of the studied films does not change appreciably. Based on distribution of the local piezoresponse after poling in Figure 9(b), the difference between the positive and negative maximum $d_{33}^{+}-d_{33}^{-}$as a function of thickness is also plotted in Figure 9(d). The observed decreasing in this difference with increasing film thickness reflects the changes in peaks symmetry, probably due to the existence of an internal bias field in films with different thicknesses.

Although several studies at the nanoscale have been reported by using PFM technique, the nature and interpretation of the shift and asymmetry in $d_{33}$ observed in Figures 9(a) and 9(b) is still not well understood. The effects of PFM tip geometry on the local piezoresponse of ferroelectric films have been reported to extract information regarding the polarization profile across a ferroelectric wall. ${ }^{28}$ It was demonstrated that the magnitude of the piezoelectric coefficient $d_{33 \text {,eff }}$ is independent on the tip radius, despite the observed unexpected $d_{33 \text {,eff }}$ decrease for smaller top electrodes ${ }^{29}$ (which should remain constant irrespectively of their size). In our measurements, the tip is a metal sphere while the bottom electrode is a flat film. To understand the possible asymmetry of the hysteresis we should recall that the PFM signal typically includes two main parts: piezo and electrostatic, as described by Eq. (4) by Hong et al. in a seminal work. ${ }^{30}$ It is expected no direct influence of bottom electrode on the electrostatic effect, since the cantilever interacts with sample surface only. Naturally, the bottom electrode may affect the growth process and consequently the self-polarization. In this case, we will have an offset on PFM images and hysteresis loops, too, but this effect does not depend explicitly on the sign of a dc bias. On the other hand, application of a bias of different sign may significantly affect the shape of the 
hysteresis loop via the injection of charge carriers through the top electrode. We believe that in our case this effect is minimal since the observed PFM signal is dominated by the piezoelectric part (we use stiff cantilevers), and there is no injection effect due to the potential barrier between the tip and surface of the film. More details about the effect of the internal bias field on the asymmetry of the PFM hysteresis loops can be found elsewhere. ${ }^{31}$

Let us now discuss about the origin of the selfpolarization effect observed in the studied PZT thin films. Often, ferroelectric films prepared by different routes exhibit a self-polarization of similar magnitude to that observed by intentional poling by an external electric field. ${ }^{32}$ The selfpolarization in highly oriented ferroelectric thin films has been mainly explained in terms of a space charge associated with the Schottky barriers located at the bottom filmelectrode interface $e^{33,34}$ or due to a mechanical coupling between the ferroelectric film and substrate. ${ }^{35}$ The asymmetrical Schottky barriers appear when the bottom and upper electrodes are made of different materials, ${ }^{32,36}$ while mechanical coupling are generally associated to the presence of a compressive/tensile stress or different thermal expansion coefficients between the film and the substrate.

The space charges of ionic and electronic origin are responsible for the built-in electric field between the film and the metallic electrode. ${ }^{37}$ This internal bias field has been considered as one of the salient features of self-polarization effect. In principle, it may be expressed as $E_{b i}=e N_{d} W / \varepsilon$ (for $h>2 W$ ) and $E_{b i}=e N_{d} h / 2 \varepsilon$ (for $h<2 W$ ), where $e$ and $N_{d}$ are the elementary charge and concentration of charge carriers, respectively, $W$ is the width of the depletion layer, and $h$ the film thickness. Assuming that the induced polarization is proportional to the average internal bias field, we expect a linear polarization decrease with thickness $h$ for $h \leq W$. For $h>W$ it is assumed that $P \propto h^{-1}$, as previously observed for PZT films prepared by sol-gel technique. ${ }^{32}$ To find out the polarization distribution across the film thickness, PFM measurements should be done destructively as previously proposed. ${ }^{38}$ As there is no direct method to access the polarization distribution across the thickness, the depletion model requires further justification. On the other hand, a perfect match between lattice parameters of the ferroelectric and of the substrate occurs in epitaxial films, leading to a strain at the interface that decays when the distance into the film bulks. ${ }^{32}$ Consequently, a linear response of the dielectric polarization as a function of the gradient strain appears along the film depth. This relationship between electric field and strain gradient is a known as flexoelectric effect. ${ }^{39,40}$ It can be described by the second term on the right-hand side of the electric polarization written as $P_{i}=d_{i j k} \sigma_{j k}+\mu_{i j k l}\left(\partial \varepsilon_{j k} / \partial x_{l}\right)$, where $d_{i j k}$ is the third-rank tensor piezoelectric coefficient, $\sigma_{j k}$ is the stress component, $\mu_{i j k l}$ is the fourth-rank tensor flexoelectric coefficient, and $\partial \varepsilon_{j k} / \partial x_{l}$ is the strain gradient. ${ }^{38,39}$

Taking into account the scenario described above, the main mechanisms responsible for the self-polarization effect in highly oriented films can be excluded as the mechanisms responsible for the same effect observed in PZT thin films studied in the present work. The linear increasing at the microstrain observed in Figure 2(a) with the glancing angle $\theta$ increasing is indicative that gradient strain along the film depth is small and does not increase substantially near the film-electrode interface, differently from that observed for highly oriented films. ${ }^{32}$ This result suggests that mechanical coupling between film-substrate, via flexoelectric effect, most probably cannot be the responsible for the selfpolarization observed in the studied films. In addition, the observed thickness behavior for $P_{i}$ shown in Figure 9(c) is essentially different from those observed in the literature for highly oriented PZT films, where $\left|P_{i}\right|$ decreases as the film thickness increases, indicating that the films are polarized by an internal bias field near the bottom film-substrate interface. ${ }^{7}$ At the films studied in the present work, $\left|\mathrm{P}_{\mathrm{i}}\right|$ increases with film thickness increasing suggesting that Schottky barriers cannot be a mechanism responsible for the selfpolarization effect for studied PZT films with no preferential orientation. ${ }^{27}$ The fact that $\left|P_{\mathrm{i}}\right|$ increases with increasing film thickness indicates that our films are polarized by an internal bias electric field strongly dependent of the film thickness, within the thickness range studied. Thus, we can exclude the effect of space charges located at the film-substrate interface and mechanical coupling between film-substrate as main mechanisms responsible for the self-polarization effect in the studied PZT films. Although the usual mechanisms to explain the self-polarization effect were excluded, the there is no conclusive evidence about the nature of the selfpolarization effect in the studied films. Further experiments are in progress to understand the origin of this phenomenon in the PZT thin films studied in the present work.

\section{CONCLUSIONS}

In summary, the kinetics of phase transformation of $\mathrm{Pb}\left(\mathrm{Zr}_{0.50} \mathrm{Ti}_{0.50}\right) \mathrm{O}_{3}$ thin films prepared by polymeric precursor method was studied in this work. The pyrolysis temperature at $300^{\circ} \mathrm{C}$ for $30 \mathrm{~min}$ defines the optimal condition for the suppression of the pyrochlore phase in the PZT thin films. The texture of the pyrochlore-free PZT films changes along the thickness leading to a small (100)-orientation tendency near the film-substrate interface. Finally, we demonstrate the existence of a self-polarization effect at both macro- and nanoscales for the studied PZT thin films. Unlike that observed for oriented PZT films, where Schottky barriers and/or mechanical coupling near the film-substrate interface are the mechanisms responsible for the selfpolarization effect, the self-polarization increase with increasing film thickness from 200 to $710 \mathrm{~nm}$ in our films suggests that these mechanisms are not primarily responsible for the observed self-polarization effect in the present work.

\section{ACKNOWLEDGMENTS}

We would like to express our gratitude to the Brazilian agencies FAPESP (Processes Nos. 2007/08534-3 and 2010/ 16504-0), CNPq (Research Grant No. 307607/2009-7 and Project No. 490436/2011-0), and CAPES (Project AUXPEPNPD, No. 2392/2009) for their financial support. Center for Research on Ceramic and Composite Materials (CICECO) of the University of Aveiro is acknowledged for the support. 
${ }^{1}$ B. Jaffe, W. R. Cook, and H. Jaffe, Piezoelectric Ceramics (Academic, New York, 1971), p. 146.

${ }^{2}$ M. Miyake, J. F. Scott, X. J. Lou, F. D. Morrison, T. Nonaka, S. Motoyama, T. Tatsuta, and O. Tsuji, J. Appl. Phys. 104, 064112 (2008).

${ }^{3}$ J. F. Scott and C. A. P. de Araujo, Science 246, 1400 (1989).

${ }^{4}$ V. Nagarajan, I. G. Jenkins, S. P. Alpay, H. Li, S. Aggarwal, L. SalamancaRiba, A. L. Roytburd, and R. Ramesh, J. Appl. Phys. 86, 595 (1999).

${ }^{5}$ J. F. M. Cillessen, M. W. J. Prins, and R. M. Wolf, J. Appl. Phys. 81, 2777 (1997).

${ }^{6}$ K. Sreenivas, M. Sayer, and P. Garret, Thin Solid Films 172, 251 (1989).

${ }^{7}$ O. Auciello, L. Mantese, J. Duarte, X. Chen, S. H. Rou, A. I. Kingon, A. F. Schreiner, and A. R. Krauss, J. Appl. Phys. 73, 5197 (1993).

${ }^{8}$ I. M. Reaney, D. V. Taylor, and K. G. Brooks, J. Sol-Gel Sci. Technol. 13, 813 (1998).

${ }^{9}$ M. Kakihana, J. Sol-Gel Sci. Technol. 6, 7 (1996).

${ }^{10}$ A. P. Wilkinson, J. S. Speck, A. K. Cheetham, S. Natarajan, and J. M. Thomas, Chem. Mater. 6, 750 (1994).

${ }^{11}$ E. B. Araújo and J. A. Eiras, J. Mater. Sci. Lett. 17, 833 (1998).

${ }^{12}$ H. M. Rietveld, Acta Crystallogr. 22, 151 (1967).

${ }^{13}$ A. C. Larson and R. B. Von Dreele, Los Alamos National Laboratory Report LAUR 86, 1994.

${ }^{14}$ B. H. Toby, J. Appl. Crystallogr. 34, 210 (2001).

${ }^{15}$ P. Thompson, D. E. Cox, and J. B. Hastings, J. Appl. Crystallogr. 20, 79 (1987).

${ }^{16}$ L. B. McCusker, R. B. Von Dreele, D. E. Cox, D. Louër, and P. Scardi, J. Appl. Crystallogr. 32, 36 (1999).

${ }^{17}$ K. G. Brooks, I. M. Reaney, R. Klissurska, Y. Huang, L. Bursill, and N. Setter, J. Mater. Res. 9, 2540 (1994).

${ }^{18}$ C. K. Kwok and S. B. Desu, Appl. Phys. Lett. 60, 1430 (1992).

${ }^{19}$ V. S. Tiwari, A. Kumar, V. K. Wadhawan, and D. Pandey, J. Mater. Res. 13, 2170 (1998).

${ }^{20}$ R. Lal, R. Krishnan, and P. Ramakrishnan, Br. Ceram. Trans. J. 87, 99 (1988).

${ }^{21}$ J. Lappalainen, V. Lantto, J. Frantti, and J. Hiltunen, Appl. Phys. Lett. 88, 252901 (2006).
${ }^{22}$ B. G. Kim, D. Kwon, B. Kim, and C. H. Chang, J. Korean Phys. Soc. 55, L1327 (2009).

${ }^{23}$ X. Y. Wen, J. Yu, Y. B. Wang, W. L. Zhou, and J. X. Gao, J. Appl. Phys. 108, 114103 (2010).

${ }^{24}$ P. E. Janolin, J. Mater. Sci. 44, 5025 (2009).

${ }^{25}$ Ragini, R. Ranjan, S. K. Mishra, and D. Pandey, J. Appl. Phys. 92, 3266 (2002).

${ }^{26}$ F. Xu, S. Trolier-McKinstry, W. Ren, B. Xu, Z. L. Xie, and K. J. Hemker, J. Appl. Phys. 89, 1336 (2001).

${ }^{27}$ E. C. Lima, E. B. Araújo, A. G. S. Filho, A. R. Paschoal, I. K. Bdikin, and A. L. Kholkin, J. Phys. D: Appl. Phys. 45, 215304 (2012).

${ }^{28}$ L. Tian, A. Vasudevarao, A. N. Morozovska, E. A. Eliseev, S. V. Kalinin, and V. Gopalan, J. Appl. Phys. 104, 074110 (2008).

${ }^{29}$ P. Gerber, A. Roelofs, C. Kügeler, U. Böttger, R. Waser, and K. Prume, J. Appl. Phys. 96, 2800 (2004).

${ }^{30}$ S. Hong, J. Woo, H. Shin, J. U. Jeon, Y. E. Pak, E. L. Colla, N. Setter, E. Kim, and K. No, J. Appl. Phys. 89, 1377 (2001).

${ }^{31}$ A. Gruverman, B. J. Rodriguez, R. J. Nemanich, and A. I. Kingon, J. Appl. Phys. 92, 2734 (2002).

${ }^{32}$ I. Kanno, S. Fujii, T. Kamada, and R. Takayama, Appl. Phys. Lett. 70, 1378 (1997).

${ }^{33}$ A. L. Kholkin, K. G. Brooks, D. V. Taylor, S. Hiboux, and N. Setter, Integr. Ferroelectr. 22, 525 (1998)

${ }^{34}$ V. P. Afanasjev, A. A. Petrov, I. P. Pronin, E. A. Tarakanov, E. Ju. Kaptelov, and J. Graul, J. Phys.: Condens. Matter 13, 8755 (2001).

${ }^{35}$ A. Gruverman, B. J. Rodriguez, A. I. Kingon, R. J. Nemanich, A. K. Tagantsev, J. S. Cross, and M. Tsukada, Appl. Phys. Lett. 83, 728 (2003).

${ }^{36}$ M. Dawber, K. M. Rabe, and J. F. Scott, Rev. Mod. Phys. 77, 1083 (2005).

${ }^{37}$ A. K. Tagantsev and G. Gerra, J. Appl. Phys. 100, 051607 (2006).

${ }^{38}$ X. M. Lu, F. Schlaphof, S. Grafström, C. Loppacher, L. M. Engb, G. Suchaneck, and G. Gerlach, Appl. Phys. Lett. 81, 3215 (2002).

${ }^{39}$ A. K. Tagantsev, Phase Transitions 35, 119 (1991).

${ }^{40}$ W. Ma, Funct. Mater. Lett. 1, 235 (2008). 